\title{
Study on Descriptors of Acoustic Emission Signals Generated by Partial Discharges under Laboratory Conditions and in On-Site Electrical Power Transformer
}

\author{
Michał KUNICKI, Andrzej CICHOŃ, Sebastian BORUCKI \\ Institute of Electrical Power and Renewable Energy \\ Opole University of Technology \\ Prószkowska 76, 45-758 Opole, Polska; e-mail: a.cichon@po.opole.pl
}

(received February 12, 2016; accepted March 14, 2016)

\begin{abstract}
An acoustic emission method (AE) is widespread and often applied for partial discharge (PD) diagnostics, mainly due to its ease of application as well as noninvasiveness and relatively high sensitivity. This paper presents comparative analysis of $\mathrm{AE}$ signals measurement results archived under laboratory conditions as well as on-site actual AE signals generated by inside PDs in electrical power transformer during its normal service. Three different PD model sources are applied for laboratory research: point to point, multipoint to plate and surface type. A typical measuring set up commonly used for on-site transformer PD diagnostics is provided for the laboratory tasks: piezoelectric joint transducer, preamplifier, amplifier and measuring PC interface. During the on-site research there are three measuring tracks applied simultaneously. Time domain, time-frequency domain and statistical tools are used for registered AE signals analysis. A number of descriptors are proposed as a result of the analysis. In the paper, attempt of AE signals descriptors, archived under laboratory condition application possibilities for on-site $\mathrm{PD}$ diagnostics of power transformers during normal service is made.
\end{abstract}

Keywords: acoustic emission; partial discharge; transformer diagnostics.

\section{Introduction}

An acoustic emission phenomenon is wide spread and relatively well described in a contemporary scientific literature (BoczAr et al., 2012; BOLIN, 1979; Pierzga et al., 2013; Pollock, Stephens, 1971). Many different sources of AE are known and the most common of them are e.g. crystal structure defects motions in solids, cracks and micro cracks forming and shifting (Wotzka, Lusa, 2015), local environment motions combined with internal friction, chemical reactions, some biological process and partial discharges. Nowadays the AE phenomenon is willingly applied in numerous science areas, especially in technical diagnostics, for machines and devices condition testing purposes (Cichoń et al., 2011; 2012; Grossmann, FESER, 2001; MENON et al., 2001; NADERI et al., 2007; SHARKAWY et al., 2005; ZHANG et al., 2007), as well as for construction structures examinations. A partial discharge diagnostics in a high voltage electrical apparatus is one of the fundamental applications of the EA method (BŁACHOWICZ et al., 2016; FARAG et al.,
1999; GARCIA-COLON et al., 2004; Lin et al., 2005). A PD issue is known as one of the most important and constant aspects of a high voltage electrical equipment insulation systems diagnostics (FRĄCZ et al., 2015; LopATKIEWICZ et al., 2012). Although a single PD source AE is represented by a discrete signal, in insulation systems there usually multi PD sources appear. As an result a summed sequence of single pulses is received by a measuring system, which in fact is read as a continuous emission signal. Not only an early detection of PD defects is highly desired presently, but also its localization and intensity estimation, especially according to a reliable and continuous electrical energy supply, as well as a save exploitation of a such primary electrical power apparatus as power transformers.

Many different PD detection and analysis methods based on a different physical phenomena accompanying a PD are presently known (SzMECHTA et al., 2011; Witos, GACEK, 2008; WotZKA et al., 2010; 2011; ZMARZŁY, NAGI, 2014). Apart from AE method, there may be pointed such main methods as a spectrophotometry method based on light emission (DENG et al., 
2001; FrĄCZ et al., 2012; 2013; FrĄCZ, 2013; WANG et al., 2006), thermovision method, based on heat emission, dissolved gas analysis (DGA) method, based on chemical reactions results, electrical method, based on electrical capacitance changes, transient earth voltage (TEV), based on electromagnetic radiation in high frequency range, and ultra-high frequency (UHF) method, based on radio frequency radiation. From among all those techniques only an electrical method is a PD direct measurement method, which means it delivers exact information about testing phenomena, i.e. an apparent charge value. All other mentioned methods are indirect measurement techniques, so measured quantities are proportional for measuring phenomena properties. There under those methods application supports PD intensity and apparent charge value survey estimation only. Nevertheless, according to a PD diagnostics an ability of on-line application, during apparatus normal service is highly essential. An AE method may be characterized by its completely non-invasiveness, and ease of application as well as relatively high sensitivity level, quite good background noise resistance level and simple measuring methodology. Furthermore, a PD source localization may be applied using an AE method, e.g. a triangulation technique. An AE measurement procedure requires the adequate amount of sensors to be placed properly on the outer wall of the tested apparatus, and allows an $\mathrm{AE}$ signals registration for a further analysis. In most cases the testing apparatus does not need to be taken out of service and a high voltage access is also not needed, as a result the highest safety level for apparatus and testing team is provided by the method.

A lot of model PD single and multi source identification ability under laboratory conditions have been proven by various different research that have been proceeded so far. Numerous partial discharge AE signal descriptors have been also aimed. The main purpose of the presented research is an on-site power transformers PD identification ability endeavor during its normal service, using AE method. Some on-site useful AE signal descriptors as well as a clear measuring methodology and results interpretation are to be proposed in the paper.

\section{Research methodology}

\subsection{Laboratory measurements}

The presented research has been proceeded in the high voltage techniques laboratory of Electrical Power and Renewable Energy institute at the Opole University of Technology. AE signals emitted by a PD source immersed in used mineral transformer insulation oil have been the research subject. Following spark gap configurations for PD source modeling have been selected for the measurements: point to point, multi- point to plate and surface type. Such a PD source models choice has allowed to simulate the most common insulation faults of electrical power transformers, e.g. point to point - a PD generated by a single insulation fault of two neighboring turns of windings, multi-point to plate - a PD generated between multi-point winding insulation faults and a grounded flat surface (tank, shielding), surface type - a PD generated on a boundary of a solid and liquid dielectrics (paper - oil). For every selected spark gap configuration an ignition $U_{i}$ and breakdown $U_{b}$ voltage levels have been experimentally defined. An electrical method with its apparent charge and voltage level dependency curves has been used for the ignition voltage appointments, whereas breakdown voltage levels have been defined after sequence of every spark gap breakdown tests, according to IEC60156. AE signals registrations have been proceeded for the selected voltage levels in the range from $U_{i}$ to $U_{b}: 120$ sample signals for every selected voltage level and for every spark gap configuration, which has given over 1800 registered signals total. A model PD source has been immersed in a steel tank filled with a transformer insulation oil. Physical Acoustics Corporation (PAC) WD-AH17 piezoelectric joint sensor has been used for measurements, and installed on the outer oil tank wall. The measuring set up has been complemented by the PAC preamplifier 2/4/6 and amplifier AE2 and Acquitek interface CH3160 installed on a PC (Fig. 1). A sampling rate of the measuring interface has been set to $1 \mathrm{MHz}$, which has allowed to register signals in the range from $20 \mathrm{kHz}$ to $500 \mathrm{kHz}$.

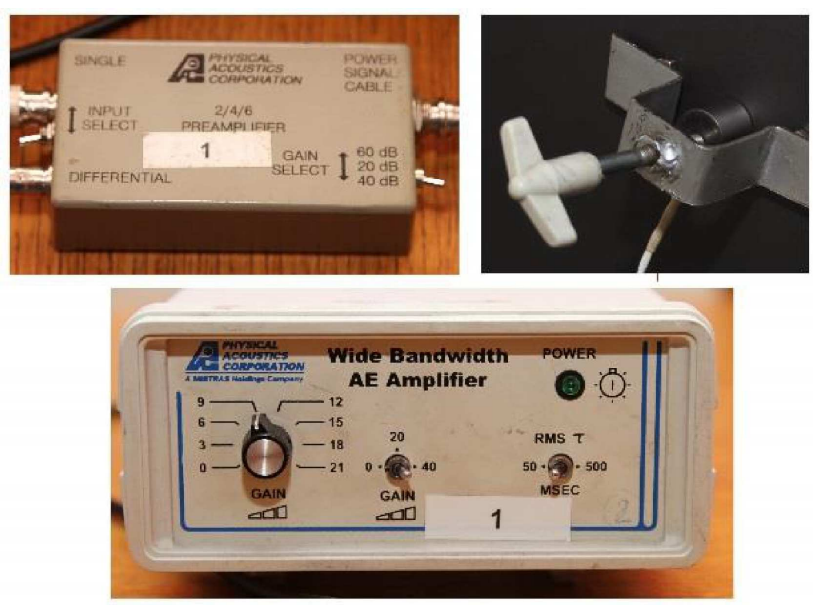

Fig. 1. Measuring instruments - general view.

A constant clamp force and repeatable sensor and tank coupling have been achieved by a dedicated magnetic grip applied additionally for measurements. A total voltage gain of the measuring track has been set to $89 \mathrm{~dB}$, and it has not been adjusted during all measurements. A PD source as well as the sensor positions have been not modified during research. Absolute distance between sensor and PD source has amounted 
$0.2 \mathrm{~m}$, which corresponds about $0.144 \mathrm{~ms}$ for the average velocity of sound in transformer mineral insulation oil which is $1390 \mathrm{~m} / \mathrm{s}$ (Fig. 2). Registered signals have been synchronized with voltage supply and measuring window has been set to $20 \mathrm{~ms}$, which is as much as one $50 \mathrm{~Hz}$ voltage supply period.

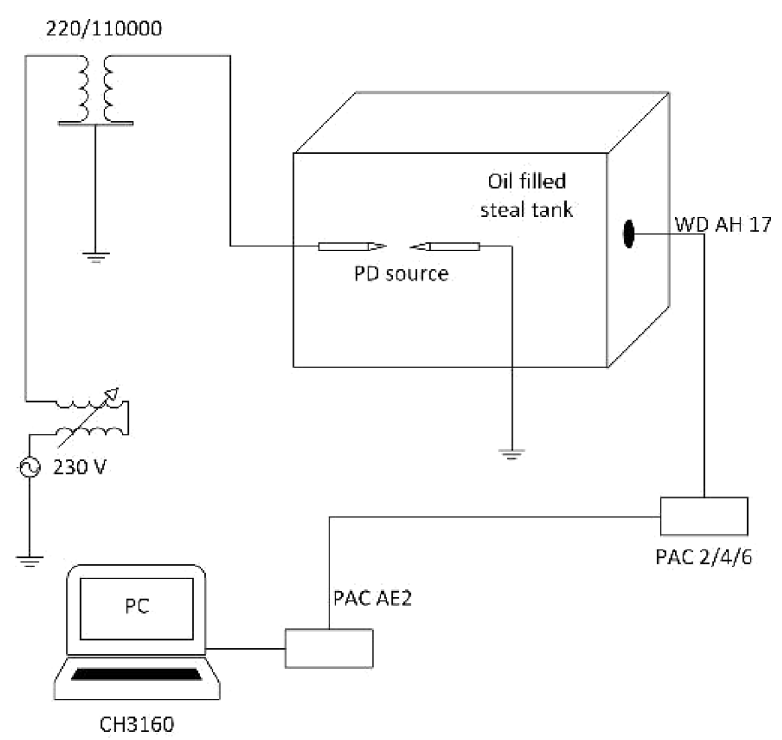

Fig. 2. Layout of the laboratory measuring set-up.

Post-measurement gathered data analysis based on Matlab software, including approximation, statistic methods and time and time-frequency domain analysis have been applied. The fast Fourier transform (FFT) has been used for frequency domain analysis, including amplitude spectrum and power density spectrum, while the short time Fourier transform (STFT) had been used for time-frequency domain analysis as well as for spectrograms achievement. Further statistical analysis of the achieved spectrums has been proceeded with selected descriptors:

a. Maximum value $E_{\max }$ of the spectrum,

b. Root mean square value (RMS) $E_{r m s}$ of the spectrum:

$$
E_{\mathrm{rms}}=\sqrt{\int_{f_{1}}^{f_{2}} E^{2}(f) \mathrm{d} f / \int_{f_{1}}^{f_{2}} \mathrm{~d} f},
$$

c. Peak factor $W_{p}\{E(f)\}$ :

$$
W_{\mathrm{p}}\{E(f)\}=\frac{E_{\mathrm{max}}}{E_{\mathrm{rms}}},
$$

where $E(f)$ - amplitude spectrum or power density spectrum, $E_{\max }-$ maximum value in spectrum, $E_{\mathrm{rms}}-$ RMS value in spectrum.

d. Shape coefficient $W_{s}\{E(f)\}$ :

$$
W_{\mathrm{s}}\{E(f)\}=\frac{E_{\mathrm{rms}}}{E_{\mathrm{avg}}},
$$

where $E_{\text {avg }}$ - mean value in spectrum.
For further analysis calculated descriptors have been gathered for every selected model PD source configuration and every pointed voltage levels.

\subsection{On-site measurements}

An electrical power transformer during normal service has been selected as a real life research object. A nominal voltage levels of the transformer have been $115 / 16.5 \mathrm{kV}$. On-site measurements have been proceeded with the same instruments as had been used in laboratory: piezoelectric joint sensor, preamplifier 2/4/6, amplifier AE2 and PC equipped with measuring interface as an acquisition unit. According to the fact that three phase transformer has been tested, three measuring tracks have had to be applied simultaneously, for supporting every single phase independent measurements (Fig. 3).

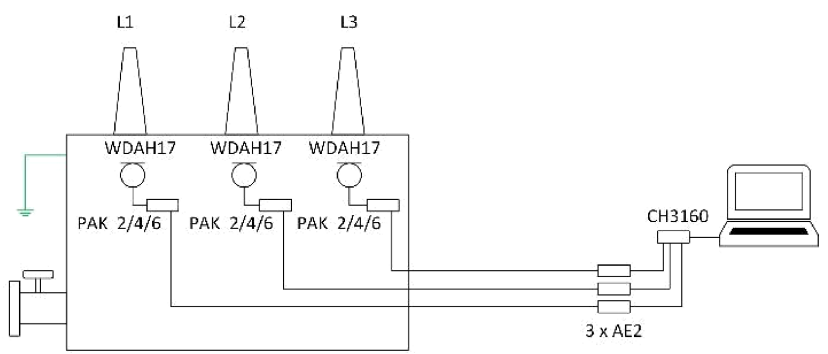

Fig. 3. Layout of the on-site measuring set-up.

AE sensors positioning procedure and arrangement has been selected experimentally, after sequence of survey tests. The final sensors positions have been pointed on grounds of the highest AE signals amplitudes, with special consideration of every phase and transformer geometry. Likewise the laboratory measurements, AE sensors have been attached to the transformer outer wall with dedicated magnetic grips for the best sensor and tank coupling (Fig. 4).

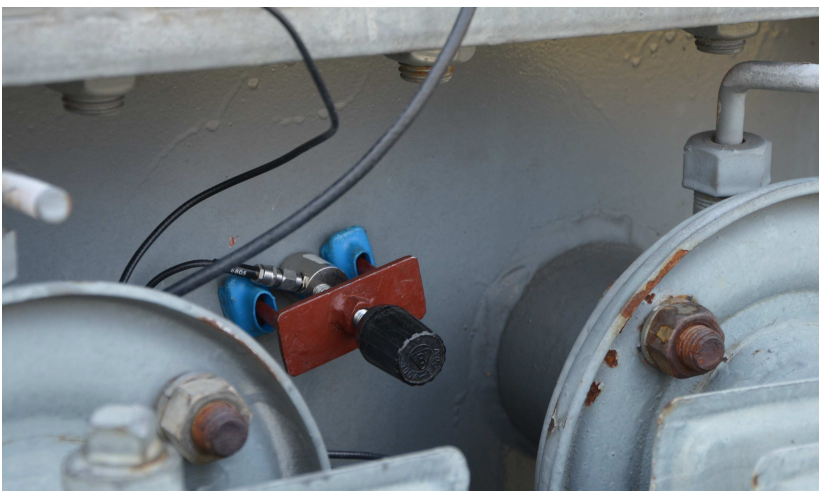

Fig. 4. AE sensor attached to the transformer - general view.

AE signals have been registered in the range from $20 \mathrm{kHz}$ to $500 \mathrm{kHz}$, and the sampling rate has been set to $1 \mathrm{MHz}$. Measuring window has been set to $20 \mathrm{~ms}$, 
like in laboratory research case, and additionally to $200 \mathrm{~ms}$, which corresponds with 1 and 10 periods of $50 \mathrm{~Hz}$ voltage supply respectively.

Unlike the laboratory research, a supply voltage level adjustment has not been possible during normal service of the testing apparatus, and all on-site measurements have been proceeded with nominal operation voltage level of the transformer. According to that fact voltage level influence on PD AE signals has not been examined. Testing object has been selected for research on the grounds of analysis of the transformer historical DGA results. According to those results very high relative growth of gas contents in oil has been discovered, which has pointed to PD activity inside the transformer tank.

\section{Results and discussion}

\subsection{Laboratory measurements}

The selected spark gaps have been configured for research tasks with particular consideration of their breakdown voltage levels. It has been desired all $U_{b}$ level should be as equal as possible. Experimentally determined $U_{b}$ levels for point to point, multi-point to plate and surface type spark gap configurations assumed $45 \mathrm{kV}, 43 \mathrm{kV}$ and $42 \mathrm{kV}$ respectively. There have been chosen relative voltage level ranges for the measurements: $0.52-0.74 U_{b}$ for point to point configuration, $0.58-0.82 U_{b}$ for multi-point to plate configuration, $0.43-0.72 U_{b}$ for surface type configuration. Analysis of the registered $\mathrm{AE}$ pulses in time domain and time-frequency domain have been the starting issue for the measurement results interpretation. Measurement results have been related with increasing supply voltage levels. Example time runs and spectrograms of captured AE signals generated by every selected model PD source for similar relative supply voltage levels have been presented in Fig. 5 .

$\mathrm{PD}$ activity in both halves of a supply voltage period has been noticed for point to point and multipoint to plate configurations, furthermore it has been observed that amplitudes of AE signals related with the first half of period have been relatively higher than those related with the second one (Fig. 6). In case of surface type configuration over $88 \%$ of all of the registered PD signals for all supply voltage levels have showed AE related only with the first half of a voltage period, which leads to the conclusion that the applied spark gap configuration generates AE only in a first positive half of the supply voltage period.

According to frequency components and energy share of individual AE signals (Fig. 7) it is easy to be noticed that the largest part of the whole energy a)
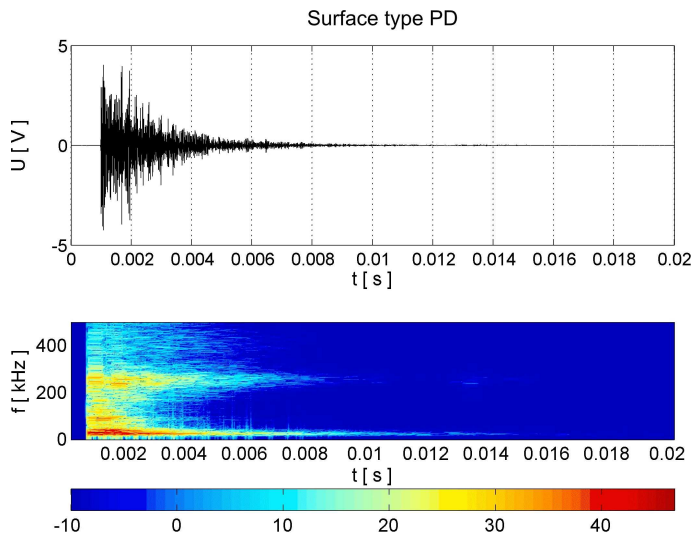

b)
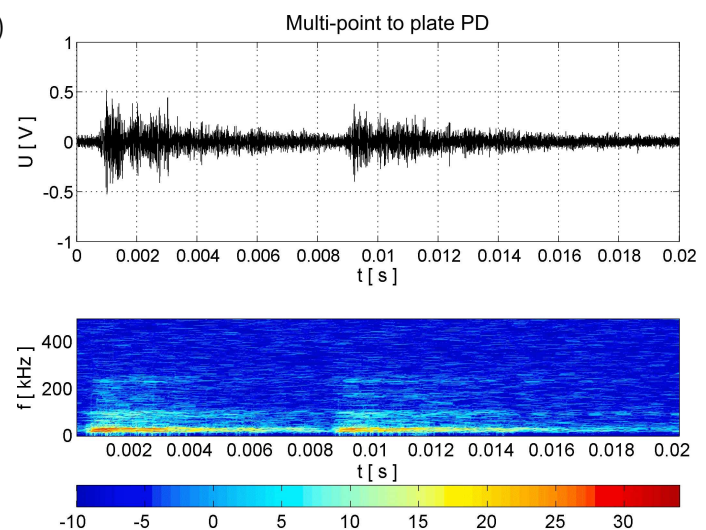

c)
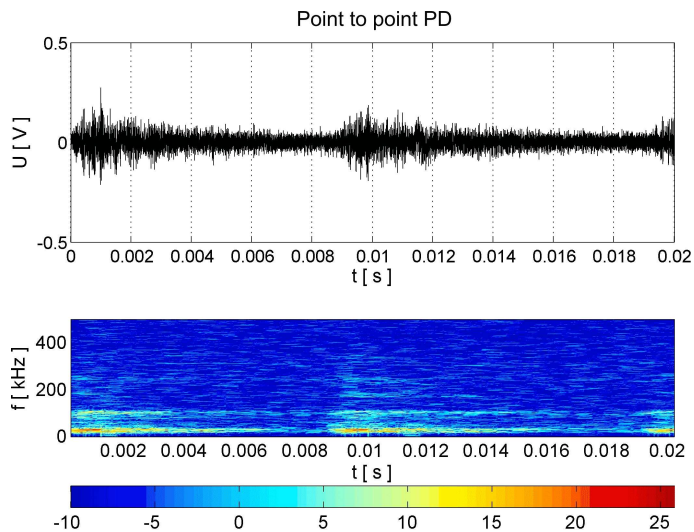

Fig. 5. Exemplary time runs and spectrogram for every spark gap configuration: a) surface type for $0.62 U_{p}$, b) multi-point to plate for $0.63 U_{p}$, c) point to point for $0.64 U_{p}$. 
a)

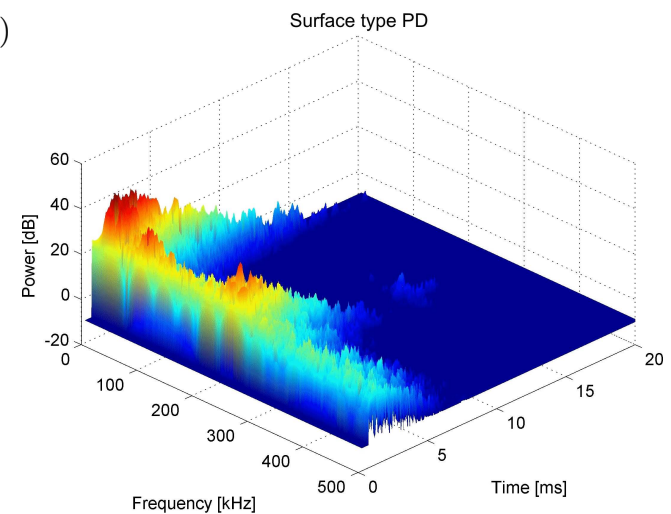

b)

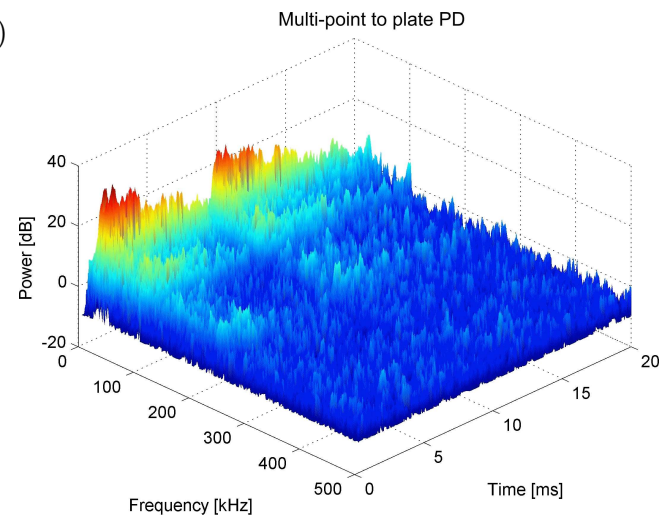

c)

Point to point $P D$

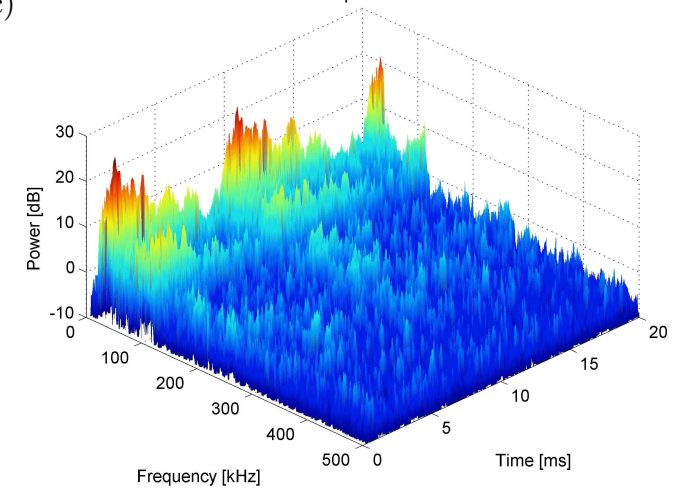

Fig. 6. Exemplary 3D spectrograms for every spark gap configuration: a) surface type for $0.62 U_{p}$,

b) multi-point to plate for $0.63 U_{p}$, c) point to point for $0.64 U_{p}$.

a)
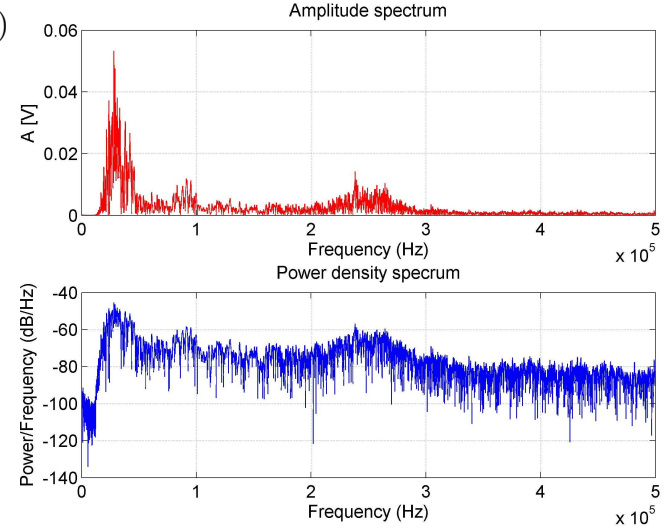

b)
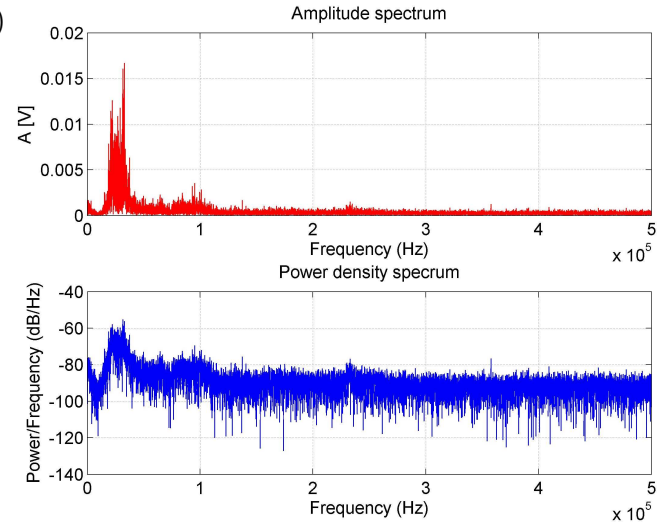

c)
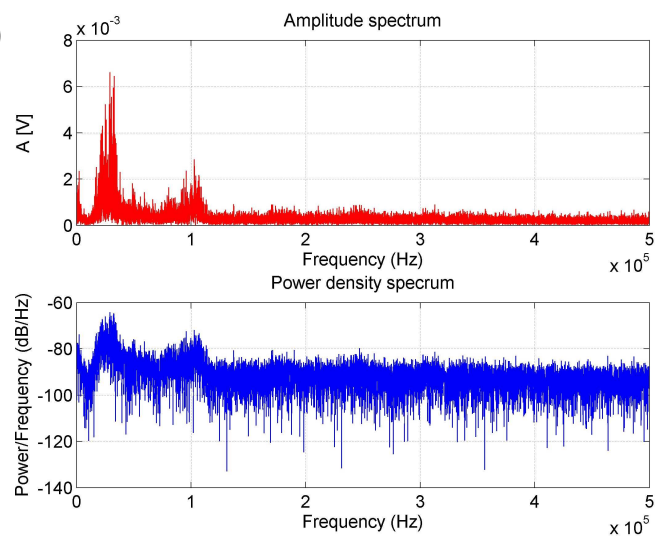

Fig. 7. Exemplary amplitude spectrum and power density spectrum for every spark gap configuration: a) surface type for $\left.0.62 U_{p}, \mathrm{~b}\right)$ multi-point to plate for $0.63 U_{p}$, c) point to point for $0.64 U_{p}$. 
is cumulated in frequency components below $100 \mathrm{kHz}$. That property has not depended on spark gap configuration and supply voltage level. Additionally, surface type PD source has generated significant part of energy for frequency components about $250 \mathrm{kHz}$. In case of other PD source configurations higher frequency components generally have not been above a background noise.

The next step of the research has been an evaluation of indicated descriptors for both amplitude and power density spectrum of analyzed AE signals. Descriptors have been defined for every registered signal for every spark gap configuration and selected supply voltage level. Mean values of every descriptor have been defined for voltage levels. A high level of coherence and reproducibility have been noticed for all achieved results, none of any descriptor relative deviation has exceeded $6 \%$. Mean values of pointed descriptors for every voltage level have been calculated on the grounds of $150 \mathrm{AE}$ signal measurements and the results have been presented as a function of voltage level. Collective results of AE amplitude spectrum de- scriptors analysis for all spark gap configuration have been shown in Fig. 8 .

Collective results of AE power density spectrum descriptors analysis for all spark gap configuration have been shown in Fig. 9, as well.

After detail analysis of achieved results some unique descriptors has been noticed. The most individual have seemed to be parameters related with RMS and maximum values of each spectrum. Apparent change ranges of those descriptors have been noticed as unique ones and may be treated as an individual parameter that characterize every PD model used for research. According to presented peak factor and shape coefficient results only power density spectrum descriptors may be considered as an unique quantities, because change trends in a voltage function of those have seemed to be individual and may be used as a support for every selected model PD source identification. Such trends have not been noticed with reference to amplitude spectrum descriptors, however for the lowest voltage levels (close to the ignition voltage value) some unique tendency has been seen a)

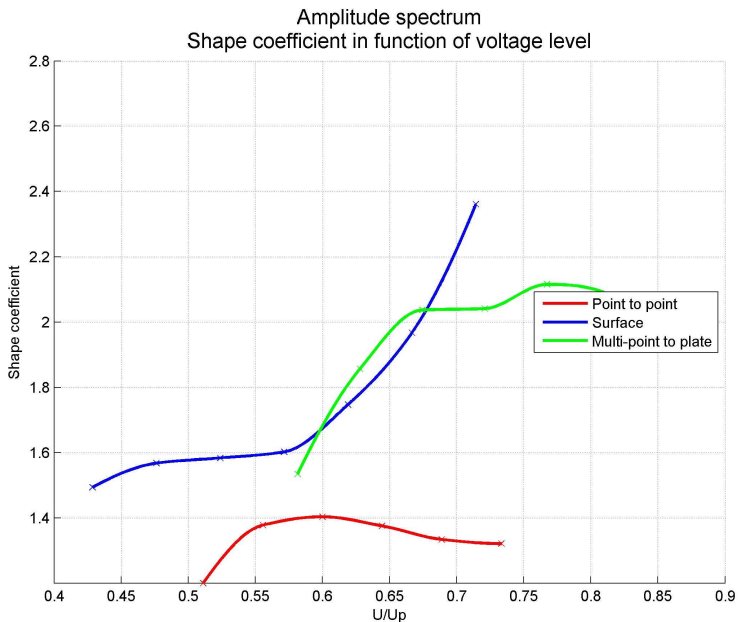

c)

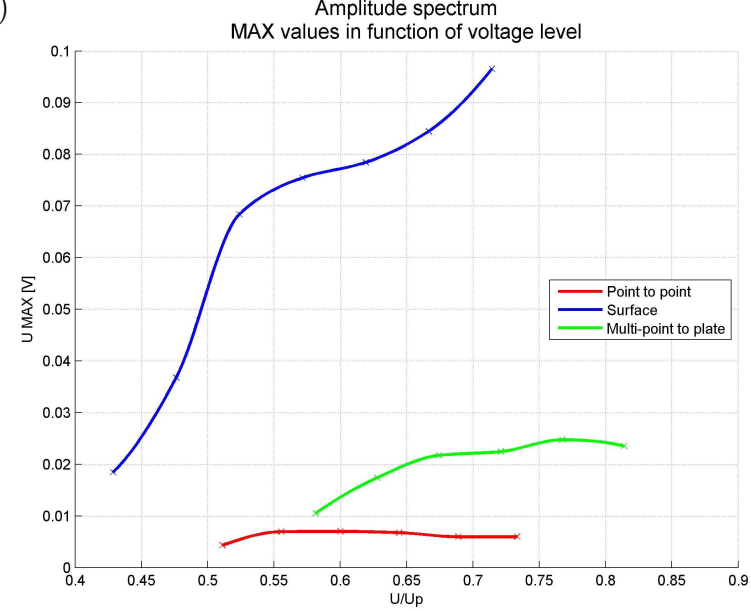

b)

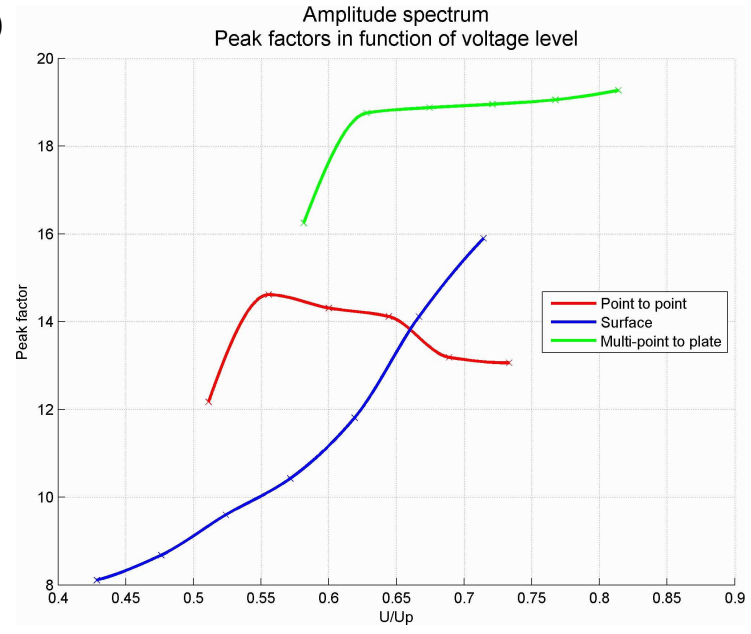

d)

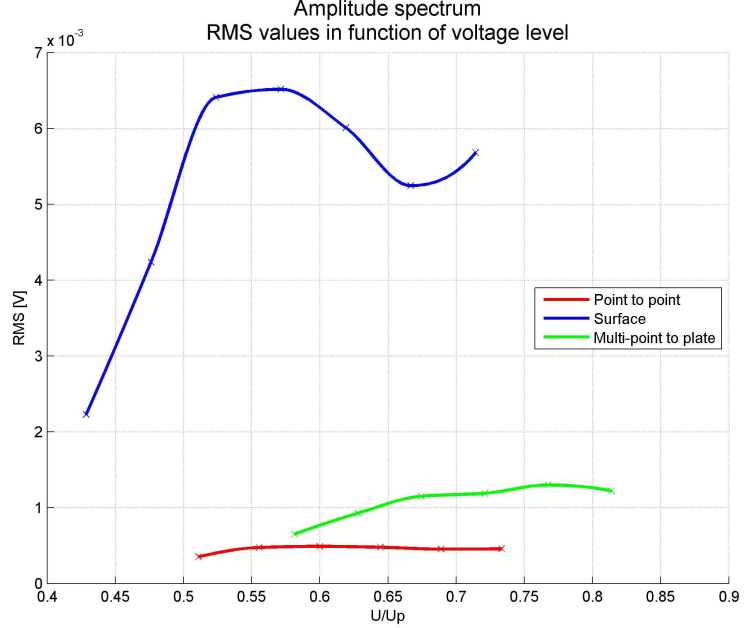

Fig. 8. Results of AE amplitude spectrum descriptors analysis: a) shape coefficient, b) peak factor, c) maximum amplitude, d) RMS value. 
a)

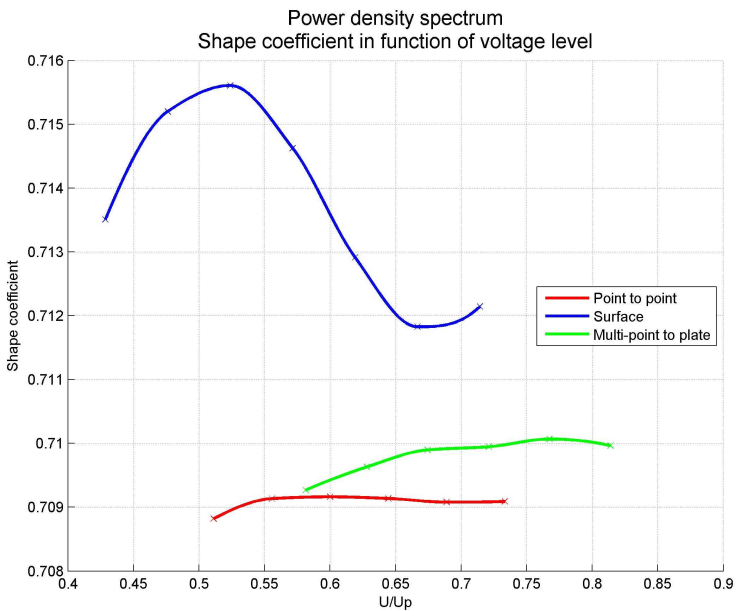

c)

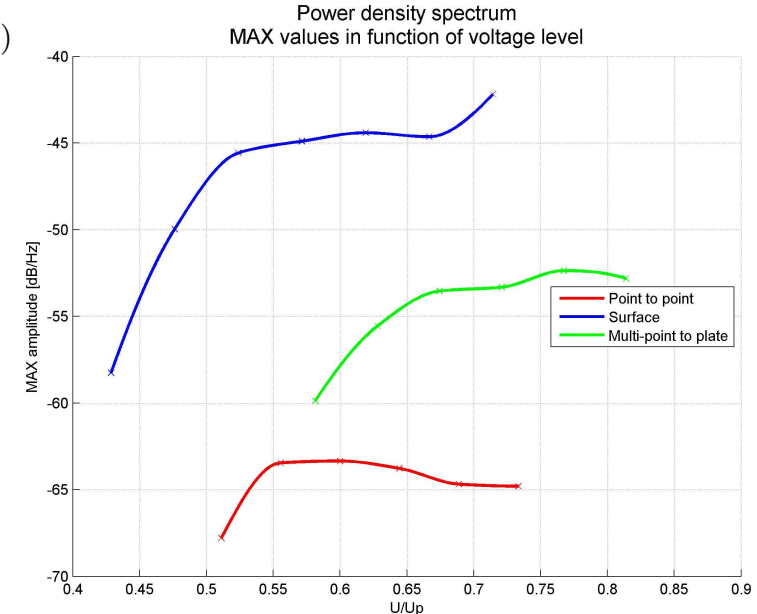

b)

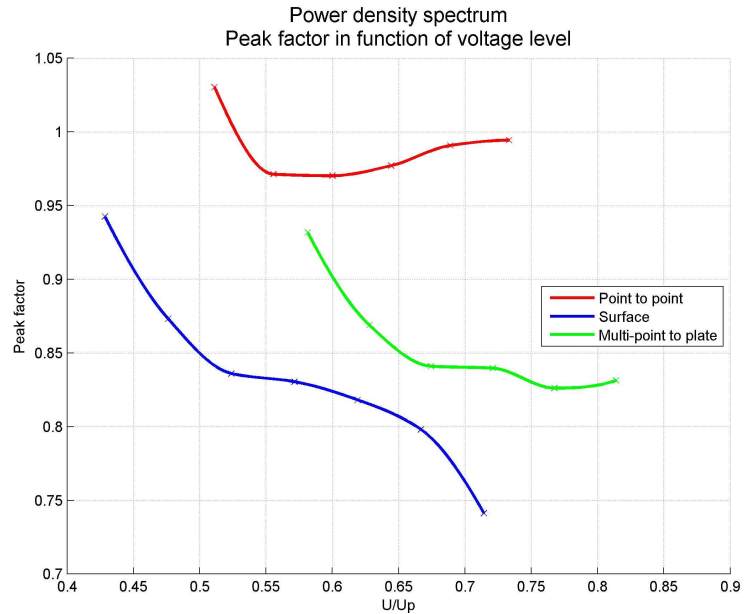

d)

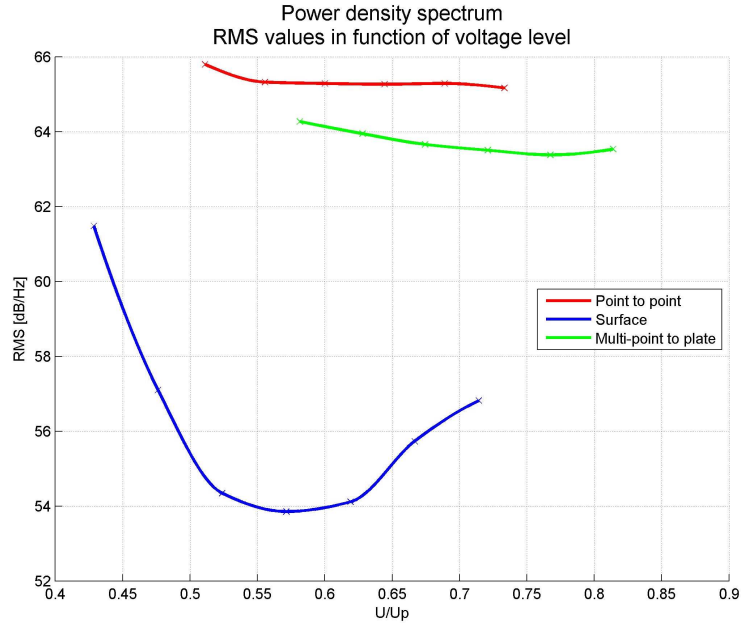

Fig. 9. Results of AE power density spectrum descriptors analysis: a) shape coefficient, b) peak factor, c) maximum amplitude, d) RMS value.

for peak factor and shape coefficient results, therefore those descriptors may be additionally used for PD source identification support also.

\subsection{On-site measurements}

A slightly different attitude is needed while a real life normal service apparatus measurements are proceeded, especially with reference to the research methodology. The main difference between laboratory measurements and on-site ones has been a supply voltage level adjustment. All of the on-site measurements have been proceeded under constant nominal working voltage level, there has been no possibility to make any adjustments. A line-to-line voltage during all tests on the transformer has been set to $118 \mathrm{kV}$. It has been also necessary to take account of much higher noise and interferences level than in laboratory environment. As mentioned above, experimental AE sensors positioning has had to be applied for electrical power transformer measurement results could be as explicit as possible. Potentially there have been three independent high voltage sources to be tested, related with the transformer three phase windings encapsulated in a common tank (Fig. 10).

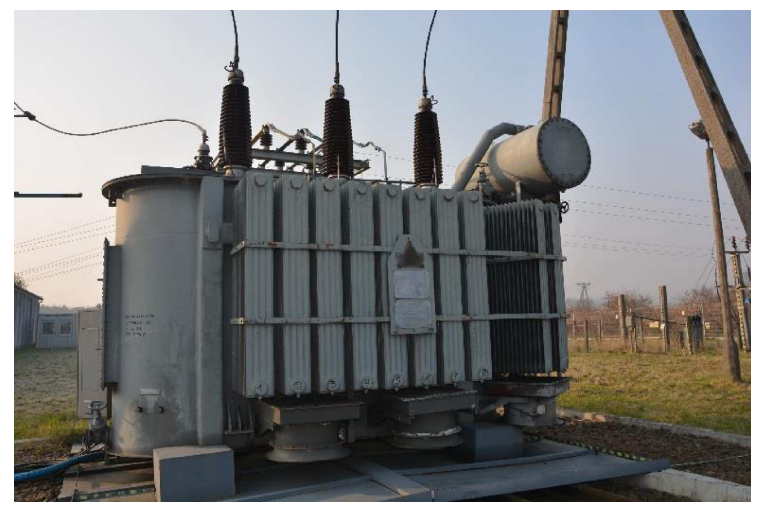

Fig. 10. Electrical power transformer $115 / 16.5 \mathrm{kV}$ used for research - general view.

The AE sensors have been finally installed under high voltage transformer bushings, on the $110 \mathrm{kV}$ side of the tank. Every sensor has been related with one phase. The distance between every sensor has been es- 
timated as $1 \mathrm{~m}$ (due to radiators installed on the outer wall of the transformer precise measurement of the distance has not been possible). Three tracks measurements have been proceeded simultaneously during all tests. Some exemplary time runs and spectrograms of registered AE signals for every phase captured within $200 \mathrm{~ms}$ (10 periods of $50 \mathrm{~Hz}$ voltage) have been presented in Fig. 11. All tracks have been synchronized and measurements have been triggered by first track pulse related with phase L1.

Periodic structures have been obviously seen at the traces, $10 \mathrm{~ms}$ intervals between serial pulses have been noticed which is related with half of $50 \mathrm{~Hz}$ voltage period - those may be interpreted as typical PD signals. The highest AE signal amplitudes have been registered by sensors positioned over phases L1 and L2, but L1 amplitudes have been twice higher than L2 ones. The L3 aimed sensor has not captured any activity that could be read as a PD signal.

Analysis of AE signals registered by sensors L1 and L2 within $20 \mathrm{~ms}$ (Fig. 12) has discovered one very important dependency: signal form the L2 sensor has been delayed about $1 \mathrm{~ms}$ toward the L1 sensor (which is about $1.4 \mathrm{~m}$ for a sound wave in oil), which has also a)
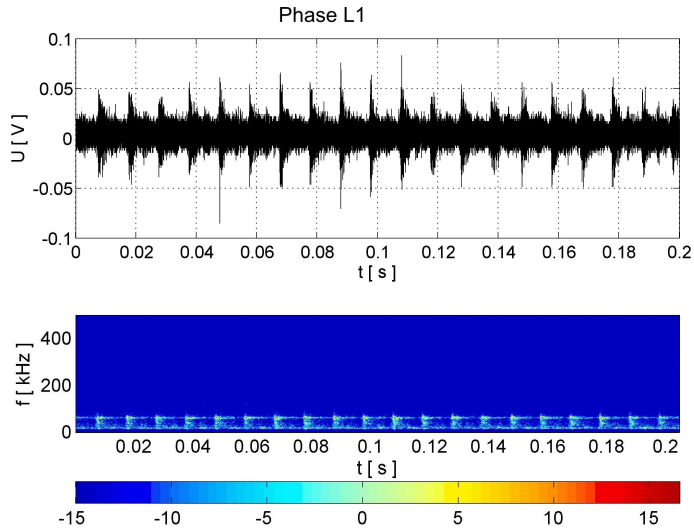

b)
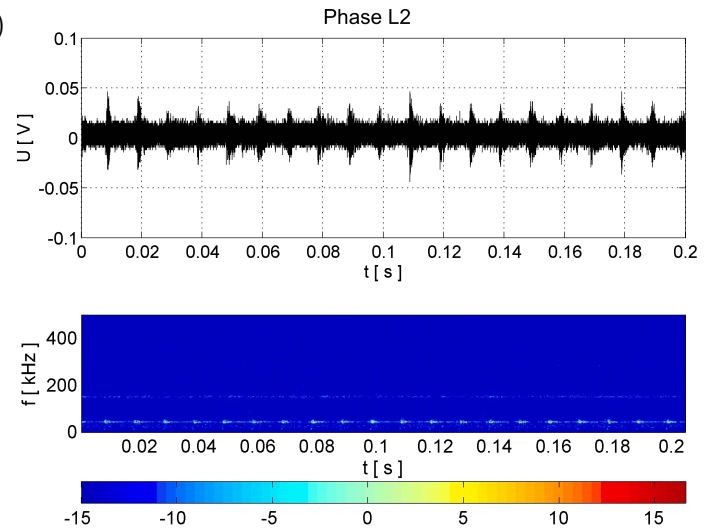

c)
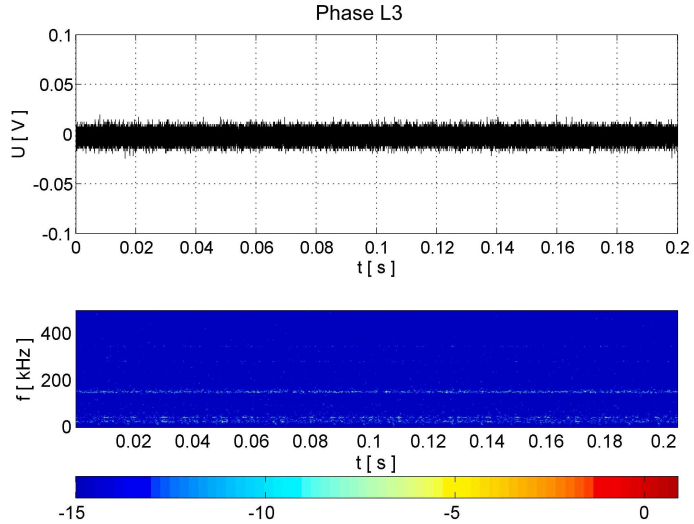

Fig. 11. Exemplary time runs and spectrograms of AE signals generated by PD inside the transformer within $200 \mathrm{~ms}$ : a) for sensor over L1 phase, b) for sensor over L2 phase, c) for sensor over L3 phase.

a)
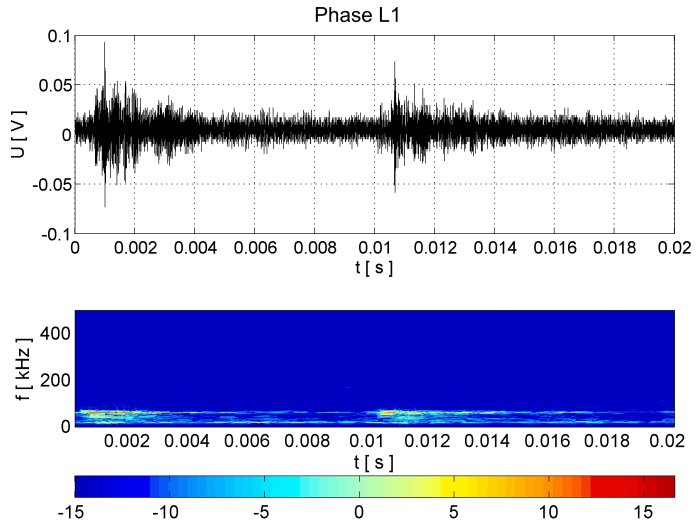

b)
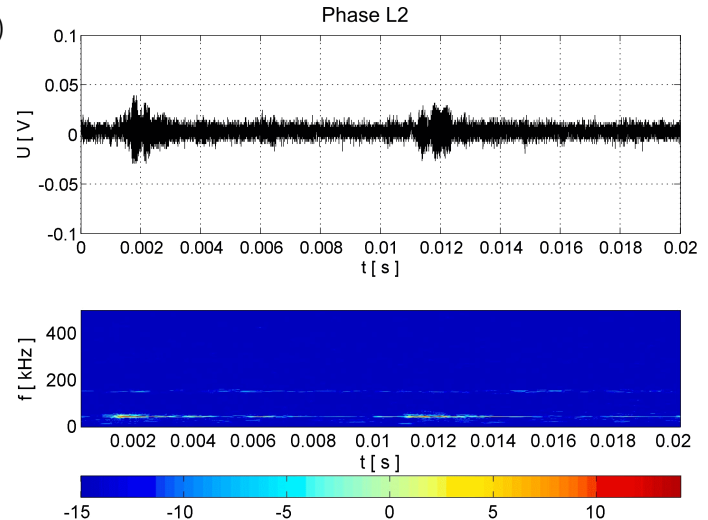

Fig. 12. Exemplary time runs and spectrograms of AE signals generated by PD inside the transformer within $20 \mathrm{ms:}$ a) for sensor over L1 phase, b) for sensor over L2 phase. 
been a trigger source. Such a dependency allow to conclude that both registered by L1 and L2 signals have been generated by the same PD source locate around the phase L1 transformer windings.

In Fig. 13 3D spectrograms of the signals presented in Fig. 12 have been showed. Strong domination of frequency components below $100 \mathrm{kHz}$ may be clearly noticed.

Likewise the laboratory measurement analysis, amplitude and power density spectrum descriptors have been determined. Exemplary spectrums of the AE signals presented in Fig. 12 have been showed in Fig. 14.

100 AE signal samples of $20 \mathrm{~ms}$ have been captured and then selected descriptors have been appointed on the grounds of them. Amplitude and power density AE signal descriptors collective results have been presented for L1 and L2 sensors in Fig. 15. L3 sensor signals have been ignored because it has not captured any activity that could be read as a PD signal.

A high level of coherence and reproducibility have been also noticed for all achieved on-site results, none of any amplitude spectrum descriptor relative deviation on a sample of 100 signals for each phase has exceeded 7\%. Similar situation has been observed for the power density spectrum descriptors, what has been presented in Fig. 16.

In the event of power density spectrum descriptors relative deviation on a sample of 100 signals for each phase has not reached $1 \%$, what confirms high reproducibility level of the measured signals. According to amplitude spectrum L1 and L2 descriptor values have varied a lot, what should have been expected after time runs and spectrograms analysis very large amplitude differences have been obviously noted. In the event of power density spectrum descriptors situations has been opposite. A high coherence level of the descriptors has been discovered, what shows a similar energy share of the same frequency components in both L1 and L2 signals. It is highly expected that both signals have been generated by similar nature sources, and taking into account all previous conclusions it has been probably the same one source. a)

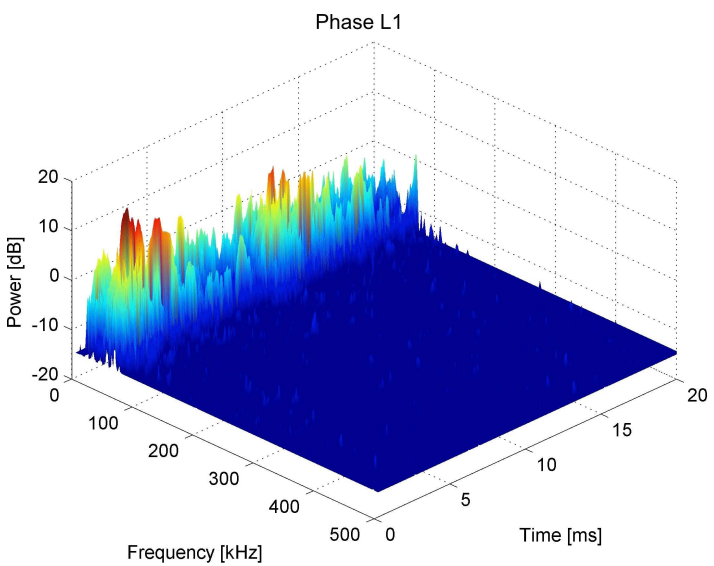

b)

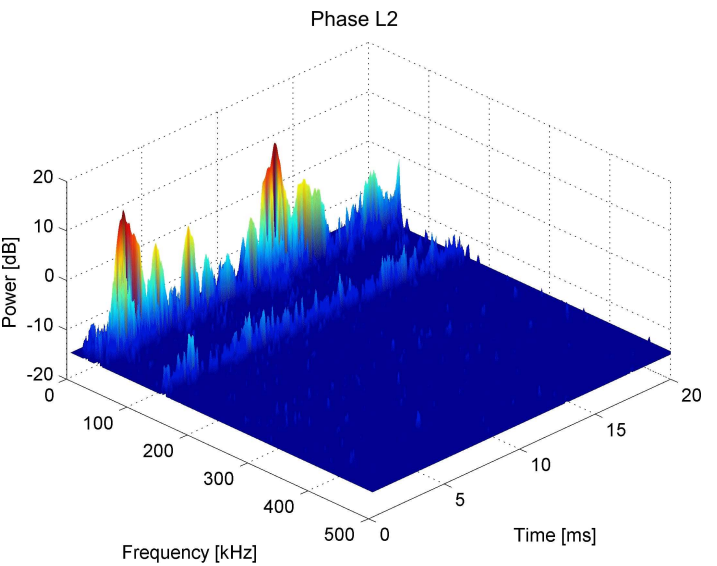

Fig. 13. 3D spectrograms of thr signals presented in Fig. 12: a) for sensor over L1 phase, b) for sensor over L2 phase.

a)
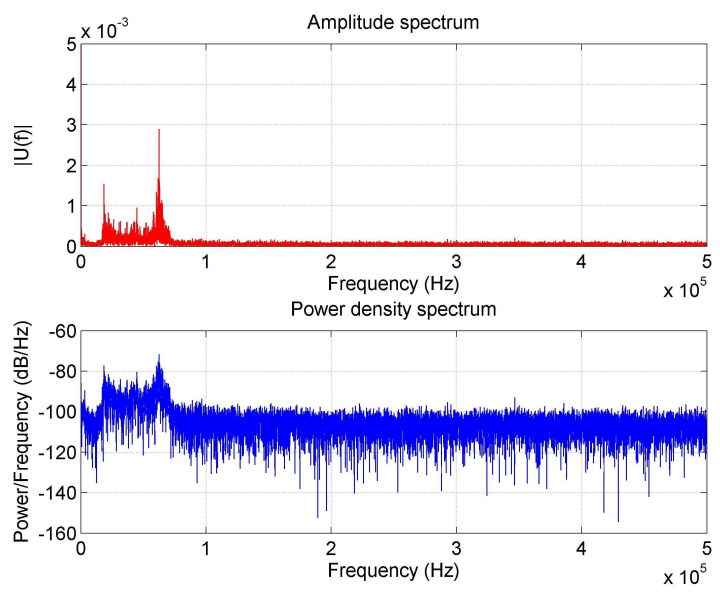

b)
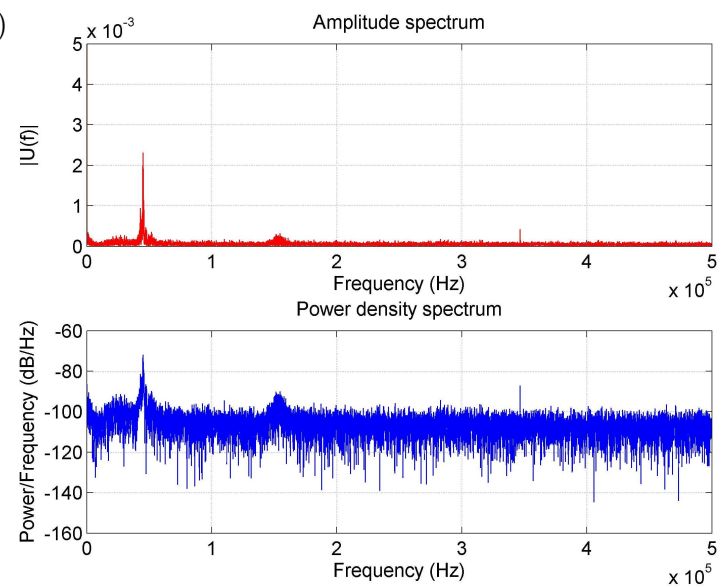

Fig. 14. Exemplary amplitude and power density spectrums of the AE signals presented in Fig. 12: a) for sensor over L1 phase, b) for sensor over L2 phase. 
a)
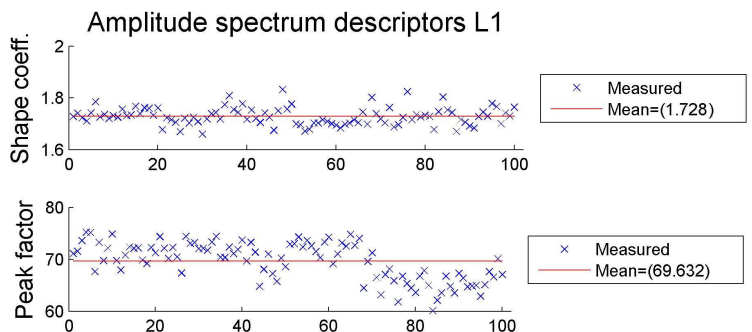

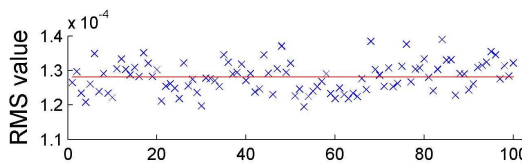

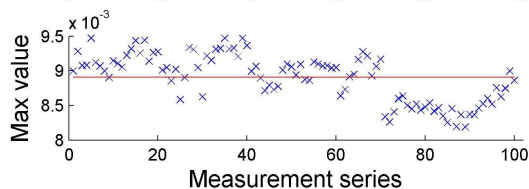
Measured $=(0.000128)$ Mean $=(0.008906)$ b)
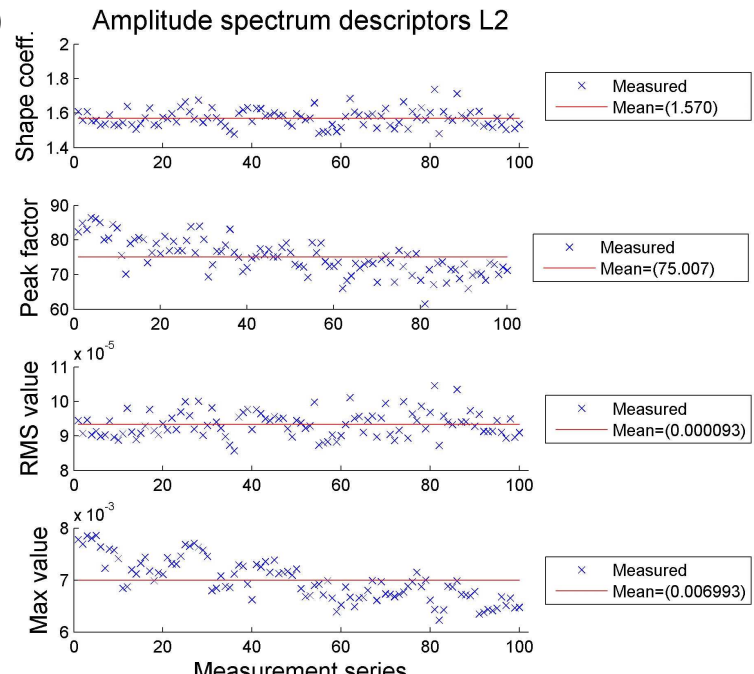

Fig. 15. Amplitude spectrum descriptors collective results of AE signals generated by PD source inside the electrical power transformer: a) for sensor over L1 phase, b) for sensor over L2 phase.

a)
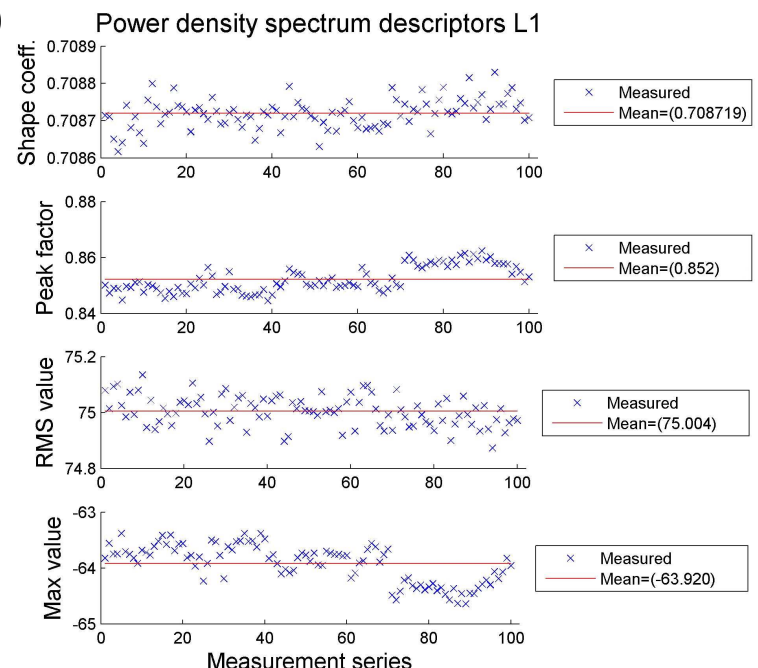

b)
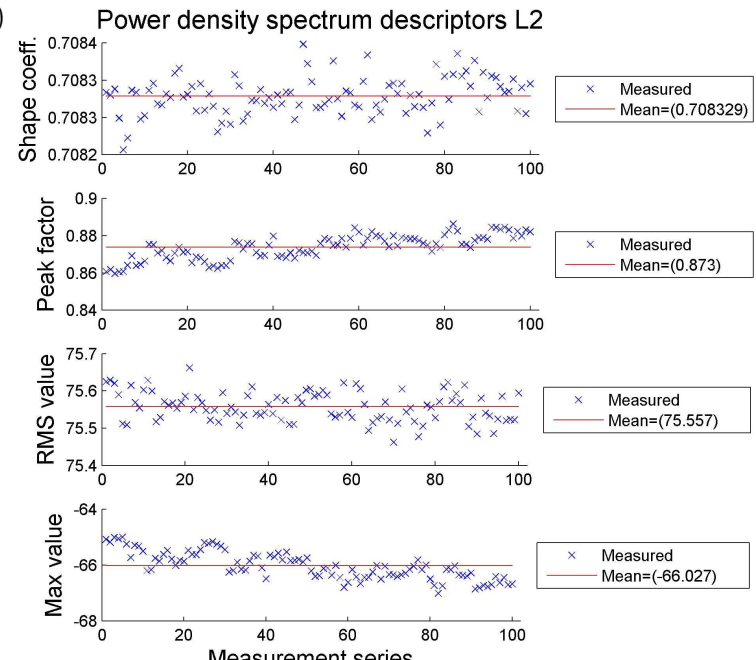

Fig. 16. Power density spectrum descriptors collective results of AE signals generated by PD source inside the electrical power transformer: a) for sensor over L1 phase, b) for sensor over L2 phase.

\section{Conclusion}

Partial discharge diagnostics in electrical power transformers is a crucial element that influences on reliable and continuous providing of electrical power supply as well as on avoiding serious faults and additional cost gain of energy distribution. Adequately early insulation faults detection provides proper procedures implementation that ensure to avoid serious problems in energy infrastructure. Wide range of contemporary signal analysis and registrations capabilities may be applied for improving and shortening technical diagnosis process. From among various commonly available PD measuring methods the most desired are noninvasive ones, and on-site applicable during apparatus normal service. It is also expected that highest sensitivity and accuracy as well as essential information about phe- nomena will be delivered by a measuring instrument. Such requirements may be very difficult or even impossible to be qualified as a part of one unrelieved measuring method. However, capability of PD analysis with particular consideration of the phenomena detection and localization for further advanced diagnostics seems to be essential. In the paper a fast, accurate and noninvasive $\mathrm{PD}$ detection and analysis methodology for electrical power transformer on-site applications has been presented. An explicit detection capability as well as a survey PD source localization of the method has been proven in the research. A PD source identification with particular consideration of its physical nature under on-site conditions still reminds an open research task. However, under a laboratory environment a model PD source identification possibility has been proven in the paper whereas a large database of sample PD signals 
related with a specific diagnosed and confirmed insulation faults in exact transformer type is needed in the event of an on-site in service transformer internal PD source identification within the considered methodology.

Authors further research tasks are planned to be focused on various on-line PD analysis methods synthesis in order to the highest precision achievement as well as measurements environment independency.

\section{Acknowledgment}

The work was co-financed from funds of the National Science Centre (NCS) as part of the OPUS programme, project no.: 2013/09/B/ST8/01736.

\section{References}

1. BŁachowicz A., Boczar T., Wotzka D. (2016), Application of a mobile system in diagnostics of power capacitors using the acoustic emission method, Insight, $\mathbf{5 8}, 2$.

2. Boczar T., Malec T., Wotzka D. (2012), Studies on infrasound noise emitted by wind turbines of large power, Acta Physica Polonica A, 122, 850-853.

3. Bolin L. (1979), A model for estimating the signal from an acoustic emission source, Ultrasonics, 17, 6770 .

4. Cichoń A., Borucki S., Wotzka D., Szmajda M. (2012), Characteristics of acoustic emission signals generated by the contacts of the selector, Acta Physica Polonica A, 122, 804-807.

5. Cichoń A., Frącz P., ZmarzŁy D. (2011), Characteristic of acoustic signals generated by operation of on load tap changers, Acta Physica Polonica A, 120, 585588 .

6. Deng J., Xiao H., Huo W., Luo M., May R., WANG A., LiU Y. (2001), Optical fiber sensor-based detection of partial discharges in power transformers, Optics \& Laser Technology, 33, 305-311.

7. Farag A.S., Shewhdi M.H., Jin X., Wang C., Cheng T.C., Dong X., Gao S., Jing W., Wang Z. (1999), On-line partial discharge calibration and monitoring for power transformers, Electric Power Systems Research, 50, 47-54.

8. Frącz P. (2013), Measurement of Optical Signals Emitted by Surface Discharges on Bushing and Post Insulator, IEEE Transactions on Dielectrics and Electrical Insulation, 20, 1909-1914.

9. Frącz P., Boczar T., Borucki S. (2013), Analysis of Optical Radiation Generated by Electrical Discharges on Support Insulator, Acta Physica Polonica A, 124, 413-416.
10. Frącz P., Boczar T., Borucki S., Cichoń A., Zmarzly D. (2012), Results of Optical Signals Analyses Emitted by Electrical Discharges Recorded with UV Camera, Acta Physica Polonica A, 122, 814-817.

11. Fracz P., Zmarzey D., Boczar T. (2015), Characteristic of Surface Partial Discharges Measured with Ultraviolet Camera, Acta Physica Polonica A, 127, 715-718.

12. Garcia-Colon V.R., Linan-Garcia R., JacoBO M.A. (2004), On-line condition assessment of high voltage current transformers, Conference Record of the IEEE International Symposium on Electrical Insulation, Indianapolis, pp. 182-185.

13. Grossman E., Feser K. (2001), Online PDmonitoring on transformers using AE techniques, Proceedings of International Conference on APTADM, Wrocław, pp. 264-268.

14. Lin D., Jiang L., Li F., Zhu D., Tan K., Wu C., Jin X., Wang C., Cheng T.C. (2005), On-line partial discharge monitoring and diagnostic system for power transformer, Tsinghua Science \& Technology, 10, 598604.

15. Lopatkiewicz R., Nadolny Z., Przybylek P. (2012), The Influence of Water Content on Thermal Conductivity of Paper Used as Transformer Windings Insulation, Proceedings of 10th IEEE International Conference on the Properties and Applications of Dielectric Materials, Bangalore, pp. 1-4.

16. Menon R., Kolambekar S., Buch N.J., RAMAMOORTY M. (2001), Correlation of acoustic emission method and electrical method for detection of partial discharges in transformer, Proceedings of 7 th IEEE International Conference on Solid Dielectrics, Eindhoven, pp. 299-302.

17. Naderi M.S., Vakilian M., Blackburn T.R., Phung B.T., NAsiri A. (2007), A hybrid transformer model for determination of partial discharge location in transformer winding, IEEE Transactions on Dielectrics and Electrical Insulation, 14, 436-443.

18. Pierzga R., Boczar T., Wotzka D., Zmarzey D. (2013), Studies on infrasound noise generated by operation of low-power wind turbine, Acta Physica Polonica A, 124, 542-545.

19. Pollock R.W.B., Stephens A.A. (1971), Waveform and frequency spectra of acoustic emissions, The Journal of Acoustical Society of America, 49, 110-114.

20. Sharkawy R.M., Fakhry S., Anis H., GhazaLY H.A. (2005), Particle detection in oil using acoustic and electrical based techniques in correlation with an inference method, Proceedings of Conference on Instrumentation and Measurement Technology, Ottawa pp. 1080-1085.

21. Szmechta M., Boczar T., Fracz P. (2011), Frequency and Time-Frequency Analysis of Acoustic Cavitation Noise in Insulating Oils, Acta Physica Polonica A, 120, 744-749. 
22. Wang P., Li B., Roman H.T., Russo O.L., Chin K., FARMER K.R. (2006), Acousto-optical PD detection for transformers, IEEE Transactions on Power Delivery, 21, 1068-1073.

23. Witos F., GACEK W. (2008), Application of the joint electro-acoustic method for PD investigation within a power transformer, The European Physical Journal Special Topics, 154, 239-247.

24. Wotzka D., Boczar T., Frącz P. (2011), Mathematical Model and Numerical Analysis of $A E$ Wave Generated by Partial Discharges, Acta Physica Polonica A, 120, 767-771.

25. Wotzka D., Lusa T. (2015), Theoretical study on the phenomena connected with cracking occurring in steel pipe based on multiphysical FE model, Journal of Mechanical Science and Technology, 29, 3225-3237.

26. Wotzka D., ZMArzŁy D., Boczar T. (2010), Numerical simulation of acoustic wave propagating in a spherical object filled with insulating oil, Acta Physica Polonica A, 118, 1272-1275.

27. Zhang H., Blackburn T.R., Phung B.T., Sen D. (2007), A novel wavelet transform technique for on-line partial discharge measurements: Part 2: On-site noise rejection application, IEEE Transactions on Dielectrics and Electrical Insulation, 14, 15-22.

28. Zmarzly D., NAGi Ł. (2014), Analysis of Ionizing Radiation Generated by Partial Discharges, Acta Physica Polonica A, 125, 1377-1379. 